\title{
Comparison of systematic coarse-graining strategies for soluble conjugated polymers
}

\author{
Christoph Scherer ${ }^{\mathrm{a}}$ and Denis Andrienko ${ }^{\mathrm{b}}$ \\ Max Planck Institute for Polymer Research, Ackermannweg 10, 55128 Mainz, Germany
}

\begin{abstract}
We assess several systematic coarse-graining approaches by coarse-graining poly(3-hexylthiophene-2,5-diyl) (P3HT), a polymer showing $\pi$-stacking of the thiophene rings and lamellar ordering of the $\pi$ stacked structures. All coarse-grained force fields are ranked according to their ability of preserving the experimentally known crystalline molecular arrangement of P3HT. The coarse-grained force fields parametrized in the amorphous melt turned out to accurately reproduce the structural quantities of the melt, as well as to preserve the lamellar ordering of the P3HT oligomers in $\pi$-stacks. However, the exact crystal structure is not reproduced. The combination of Boltzmann inversion for bonded and iterative Boltzmann inversion with pressure correction for nonbonded degrees of freedom gives the best coarse-grained model.
\end{abstract}

\section{Introduction}

Coarse-graining is a systematic way of reducing the number of degrees of freedom describing a specific physical system. A typical but by no means complete list of coarsegraining procedures includes: (i) A renormalization group analysis in the vicinity of a critical point, where degrees of freedom (e.g. spins) are blocked together [1]. (ii) The formulation of system dynamics in terms of a master equation, with the entire phase space represented by a few states [2]. (iii) Parametrization of classical force-fields, in which electronic degrees of freedom are incorporated into classical interaction potentials [3]. (iv) United-atom or coarser classical particle-based force fields, with light atoms (e.g. hydrogens) incorporated into the heavier ones [4].

Coarse-graining often relies on a certain time-scale separation: some parts of the system evolve on a significantly slower timescale, e.g., a thiophene ring rotation in a polythiophene is a much slower process than a characteristic bond vibration. In this case it is possible to combine several coherently moving atoms into a single interaction site, reducing the number of degrees of freedom to be propagated and, more importantly, obtaining a smoother potential energy landscape. Softer interaction potentials lead to less friction (faster dynamics), helping to reach ten to hundred times longer simulation times.

The coarse-graining procedure in itself involves three steps: choice of the coarsegrained degrees of freedom, identification of a merit function which quantifies the

\footnotetext{
a e-mail: christoph.scherer@mpip-mainz.mpg.de

b e-mail: denis.andrienko@mpip-mainz.mpg.de
} 
difference between the fine- and coarse-grained representations, and determination of the coarse-grained potential energy surface (PES). The entire procedure can be thought of as a projection of the fine- onto the coarse-grained PES and is therefore sensitive to the number and types of basis-functions employed in the CG representation. To perform the same statistical sampling of the coarse-grained degrees of freedom, ideally, the potential of mean force should be used as the coarse-grained interaction potential [5]. This is inherently a many-body potential which, in practice, is projected on basis functions that are used to represent the coarse-grained force-field. Existing projection schemes either try to reproduce various pair distribution functions (structure-based coarse-graining $[6,7]$ ), to match the forces $[8,5]$, to minimize the information loss in terms of relative entropy [9], or to make use of the liquid state theory [10].

While these coarse-graining techniques have been successfully applied to simple liquids (solvents) [11, 7, 12], amorphous polymer melts [13, 14, 15, 16, 6], and amorphous organic solids [4], the number of examples with more structured macromolecular systems is rather sparse. The systems with long-range order are, however, exactly the systems where coarse-grained models are needed the most: In many fields, one of the most challenging tasks is to quantify the self-organizing abilities of a material solely based on its chemical structure. For example, in polymeric organic semiconductors, self-assembled structures are lamellar arrangements of conjugated polymers, partially crystalline phases of a small-molecule donor/acceptor material, or molecular alignments at the interface between the organic layers. However, the final goal of studying the whole process of self-assembly on a computer has not been achieved so far [17].

The purpose of this work is to review the performance of existing coarse-graining techniques when applied to a complex, self-assembling, polymeric system. As a test case, we consider regioregular poly(3-hexylthiophene-2,5-diyl) (P3HT), the chemical structure of which is shown in Fig. 1. P3HT is used as a donor in bulk heterojunction solar cells $[18,19]$. In combination with PCBM as an acceptor, it yields device efficiencies of up to $4.4 \%$, which in 2005 was the highest achieved value for polymeric solar cells [20].

P3HT is an ideal case study for testing coarse-graining models of macromolecular systems: It is a polymorph with three different crystal structures, form I, I' and II $[21,22,23]$. All polymorphs show a $\pi$-stacking of the thiophene rings in $b$-direction and a lamellar ordering in $a$-direction of the unit cell. They differ in the conformation and the interdigitation of the hexyl side chains, the inclination of the backbones with respect to the $\pi$-stacking direction and the shift of successive polymer chains along the $\pi$-stacking direction. The polymorph I, for example, has a monoclinic unit cell with $a=1.60 \mathrm{~nm}, b=0.78 \mathrm{~nm}$ and $c=0.78 \mathrm{~nm}[24]$. Depending on the processing conditions, P3HT can crystallize into high aspect-ratio secondary structures, e.g., up to micrometer long nanofibres $[25,26,27,28,29]$. This can be achieved by solvating P3HT into a poor solvent or a mixture of different solvents and a slow cooling process $[26,27]$. These structures are of particular interest to achieve a good device efficiency of field-effect transistors.

P3HT has also been studied using different types of simulation techniques: classical force fields have been parametrized $[30,31]$ to study crystalline phases of P3HT [30, $32]$ and high-temperature amorphous melts [33, 34], for which the melting temperature $(490 \mathrm{~K})$ and the glass transition temperature $(300 \mathrm{~K})$ were shown to be in good agreement with experimental results [32]. However, due to limitations regarding the chain lengths and simulation times, it was not possible to observe the process of self-assembly or crystallization from solvated P3HT chains or an amorphous melt in all-atom classical MD simulations. Some ordering of the chains has been observed when cooling the amorphous melt [35]. 


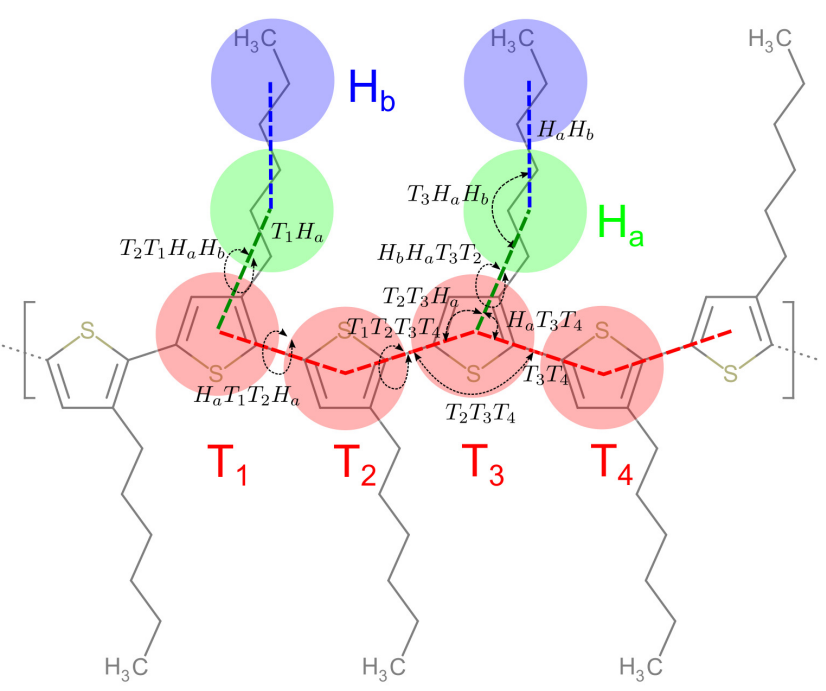

Fig. 1. Chemical structure of regioregular poly(3-hexylthiophene-2,5-diyl) (P3HT), its mapping scheme with three types of beads, $T, H_{a}$, and $H_{b}$, as well as eleven bonded degrees of freedom. Note that in the text the subscripts of the $T$ beads are omitted.

Coarse-grained models of P3HT with different resolution have also been developed where one repeat unit of P3HT is modeled either with one [36] or three coarse-grained beads [37, 38, 39, 40]. To this point, all coarse-grained models have been derived with structure-based techniques either on the basis of a high-temperature amorphous melt [37, 38], a liquid mixture of P3HT and PCBM [36] or P3HT monomers solvated in anisole (methoxybenzene) solvent [39], with some empirical refinements [40]. In all cases, the non-bonded interactions are modeled as isotropic two-body interactions and are either tabulated [37, 38, 39] or Lennard-Jones potentials [36, 40]. Regarding the three-site models, the onset of phase separation between P3HT and PCBM is observed upon cooling from the melt $[37,38]$. Simulations with nonbonded potentials obtained from the single-monomers solutions show lamellar ordering into layers [40] and an aggregation of P3HT chains with decreasing temperature [39]. The spacing of the lamellae of about $1.7-2.0 \mathrm{~nm}[39,40]$ is found to be in agreement with experimental results of $1.68 \mathrm{~nm}$ [41].

In this work, we would like to assess the quality of systematic bottom-up coarsegraining approaches when applied to a complex self-assembling system, such as P3HT. Realizing that self-assembling the systems in silico is computationally too demanding even for a coarse-grained model, we here use a much simpler criterion: a good coarse-grained model should preserve, as closely as possible, the experimentally known self-assembled structure of a polymer. Atomistic force-fields, for example, are doing extremely well in this respect [30].

The paper is organized as follows. We first describe the coarse-grained model in section 2. We then compare the resulting potentials in sections 3 and 4 . Finally, we summarize the performance of coarse-grained models in section 5 .

\section{Coarse-grained degrees of freedom}

In all studied cases, we use three types of coarse-grained beads per repeat unit of $\mathrm{P} 3 \mathrm{HT}$, representing the thiophene ring $T$, the first three, $H_{a}$, and the last three, 
$H b$, methyl groups of the hexyl side-chain, as shown in Fig. 1. This CG scheme is consistent with previous works $[37,38,39,40]$. Here, however, the $T$-bead is located at the intersection of the two $\mathrm{C}-\mathrm{C}$ bonds connecting the thiophene ring with the next and the previous thiophene unit of the P3HT chain. This center of the thiophene bead $T$ does not correspond to the center of mass (COM) of the thiophene ring. The reason for this choice of the mapping coefficients is to prevent coupling of bond and angular degrees of freedom along the thiophene backbone. The $H_{a}$ and $H_{b}$ beads are located at the COM of the methyl groups.

To determine bead positions, the linear mapping operator is used

$$
\mathbf{R}_{i}=\sum_{\alpha \in i} w_{\alpha} \mathbf{r}_{\alpha}
$$

where $\mathbf{R}_{i}$ is the position of the $i$-th CG bead, $w_{\alpha}$ are the mapping coefficients, and $\mathbf{r}_{\alpha}$ are the coordinates of atoms belonging to the bead $i$.

The masses of the CG beads are set to obtain consistency in momentum space [5]

$$
M_{i}=\left(\sum_{\alpha \in i} \frac{w_{\alpha}^{2}}{m_{\alpha}}\right)^{-1},
$$

resulting in $M_{T}=57.406 \mathrm{amu}, M_{H_{a}}=42.048 \mathrm{amu}$ and $M_{H_{b}}=43.056 \mathrm{amu} . M_{\mathrm{Ha}}$ and $M_{\mathrm{Hb}}$ are equal to the sum of the atomistic masses, whereas $M_{\mathrm{T}}$ is only $70 \%$ (end beads of the chain) and $71 \%$ (beads in the center of the chain) of the sum of the atomistic masses of one thiophene unit.

For the three-bead model, eleven bonded degrees of freedom are introduced (see also Fig. 1): three bonds, $T T, T H_{a}$, and $H_{a} H_{b}$; four angles, $T T T, T T H_{a}, H_{a} T T$, and $T H_{a} H_{b}$; and four dihedrals, TTTT, $H_{a} T T H_{a}, T T H_{a} H_{b}$, and $H_{b} H_{a} T T$, similar to the ones discussed in Refs. $[37,38,39]$. Note that, due to the directionality of the regioregular P3HT chain, it is necessary to differentiate the $T T H_{a}$ and the $H_{a} T T$, as well as the $T T H_{a} H_{b}$ and the $H_{b} H_{a} T T$ interactions.

In terms of nonbonded interactions, we have limited ourselves to pair potentials between all bead types, i.e. six pairwise interaction potentials. This is again in line with the CG models discussed in Refs. [37, 38, 39].

Selection of coarse-grained beads, corresponding degrees of freedom, and basis functions of the coarse-grained force fields completes the first coarse-graining step. In what follows we parametrize the basis functions of the coarse-grained force field using the VOTCA package [7].

\section{Bonded interactions}

We will start with the eleven bonded interactions. To parametrize the bonded potentials we use two different methods and three different setups:

1. Force matching of all degrees of freedom together (see Appendix $\mathrm{C}$ for the details of the method). In this case, 6 nonbonded interactions are parametrized simultaneously with 11 bonded interactions, i.e. 17 different tabulated interaction potentials are determined together. Parametrizations are performed in a melt at $500 \mathrm{~K}$ and in a glassy amorphous system (quenched melt) at $300 \mathrm{~K}$.

2. Boltzmann inversion (see Appendix B for details) of bond, angular, and dihedral distributions. These distributions are sampled for an isolated chain with exclusions, i.e. beads further that four bonds apart are not interacting with each other. This is often used to derive CG potentials for polymer melts, where non-bonded interactions are screened. The sampling has been performed at two temperatures, $500 \mathrm{~K}$ and $300 \mathrm{~K}$. 


\begin{tabular}{|c||c|c|c|}
\hline $\begin{array}{c}\text { system } \rightarrow \\
\mathrm{CG} \text { method } \downarrow\end{array}$ & $\begin{array}{c}\text { single chain with } \\
\text { exclusions }\end{array}$ & $\begin{array}{c}\text { single chain in a } \\
\text { solvent }\end{array}$ & amorphous melt \\
\hline \hline BI at $300 \mathrm{~K}$ & $\times$ & $\times$ & $\times$ \\
\hline BI at $500 \mathrm{~K}$ & $\times$ & & $\times$ \\
\hline FM at $300 \mathrm{~K}$ & & & $\times$ \\
\hline FM at $500 \mathrm{~K}$ & & & $\times$ \\
\hline
\end{tabular}

Table 1. Different systems and coarse-graining methods (Boltzmann inversion, BI, and force matching, FM) used to parametrize bonded interactions. The FM method here is used to parametrize nonbonded and bonded interactions simultaneously.

3. Boltzmann inversion of the distributions obtained by sampling a single chain solvated in dichlorobenzene (DCB), without any exclusions.

4. Boltzmann inversion in a melt at $500 \mathrm{~K}$ or glassy amorphous systems (quenched melt) at $300 \mathrm{~K}$.

All systems and CG schemes used to parametrize the bonded interactions are summarized in table 1 and shown in Fig. 2 (angles) and Fig. 3 (dihedrals), together with the results presented in Ref. [37, 39]. The bond length potentials are shown in the Supporting Information and are similar for all methods. All tabulated interactions, the coefficients of the Ryckaert-Belleman potentials and the bond length potentials are given in the supporting information.

Angular potentials In principle, all methods lead to comparable angular potentials. Boltzmann inverted distributions are practically the same for the amorphous melt, the isolated chain with exclusions, and a chain in a solvent. The FM potentials show a systematic shift of the potential minimum to about $20^{\circ}\left(T T H_{a}, H_{a} T T\right)$ and $10^{\circ}(T T T)$ higher values with respect to the BI potentials, both at $300 \mathrm{~K}$ and $500 \mathrm{~K}$. For $T H_{a} H_{b}$ there is no shift but FM potentials are slightly stiffer than the BI potentials. The potentials of Refs. [37, 39] are significantly softer. This can be explained by a difference in mapping schemes, as discussed in sec. 2: if the centers of mass of the thiophene rings are used as $T$-bead positions, as it is done in Refs. [37, 39], the dihedral and angular degrees of freedom are coupled, and a larger variation of the bond angle is observed. Indeed, the most pronounced differences between the potentials parametrized in this work and the ones of the $353 \mathrm{~K}$ solvent and the $550 \mathrm{~K}$ melt $[37,39]$ are observed for the TTT angular potentials: The latter are significantly broader and the potential minimum is shifted to bond angles between about $160^{\circ}-170^{\circ}$, in contrast to about $145^{\circ}-155^{\circ}$ for the potentials parametrized in this work.

Dihedral potentials The bond dihedral interactions of the different systems and parametrization methods are compared to the ones of previous studies [37, 39] in Fig. 3. One can see that all $T T H_{a} H_{b}$ BI potentials are rather similar and agree to the ones parametrized at $353 \mathrm{~K}$ and $550 \mathrm{~K}[37,39]$. The FM potentials show distinct differences and lack the additional potential maximum at $\phi=0^{\circ}$. The $H_{b} H_{a} T T$ potentials of the different systems and temperatures are also rather similar to each other. Here, the differences of the FM potentials are less distinct. In all cases, a potential maximum at about $\phi=0^{\circ}$ and $\phi= \pm 180^{\circ}$ is visible. However, the maxima at $\phi=0^{\circ}$ of the BI potentials are significantly broader and the ones parametrized at $300 \mathrm{~K}$ show an additional small dip at about $\phi=0^{\circ}$. Both $H_{a} T T H_{a}$ potentials of previous studies [37,39] have minima at $\phi=0^{\circ}$ and $\phi= \pm 180^{\circ}$. The potential minimum at $\phi= \pm 180^{\circ}$, in both cases, is deeper which corresponds to the trans 

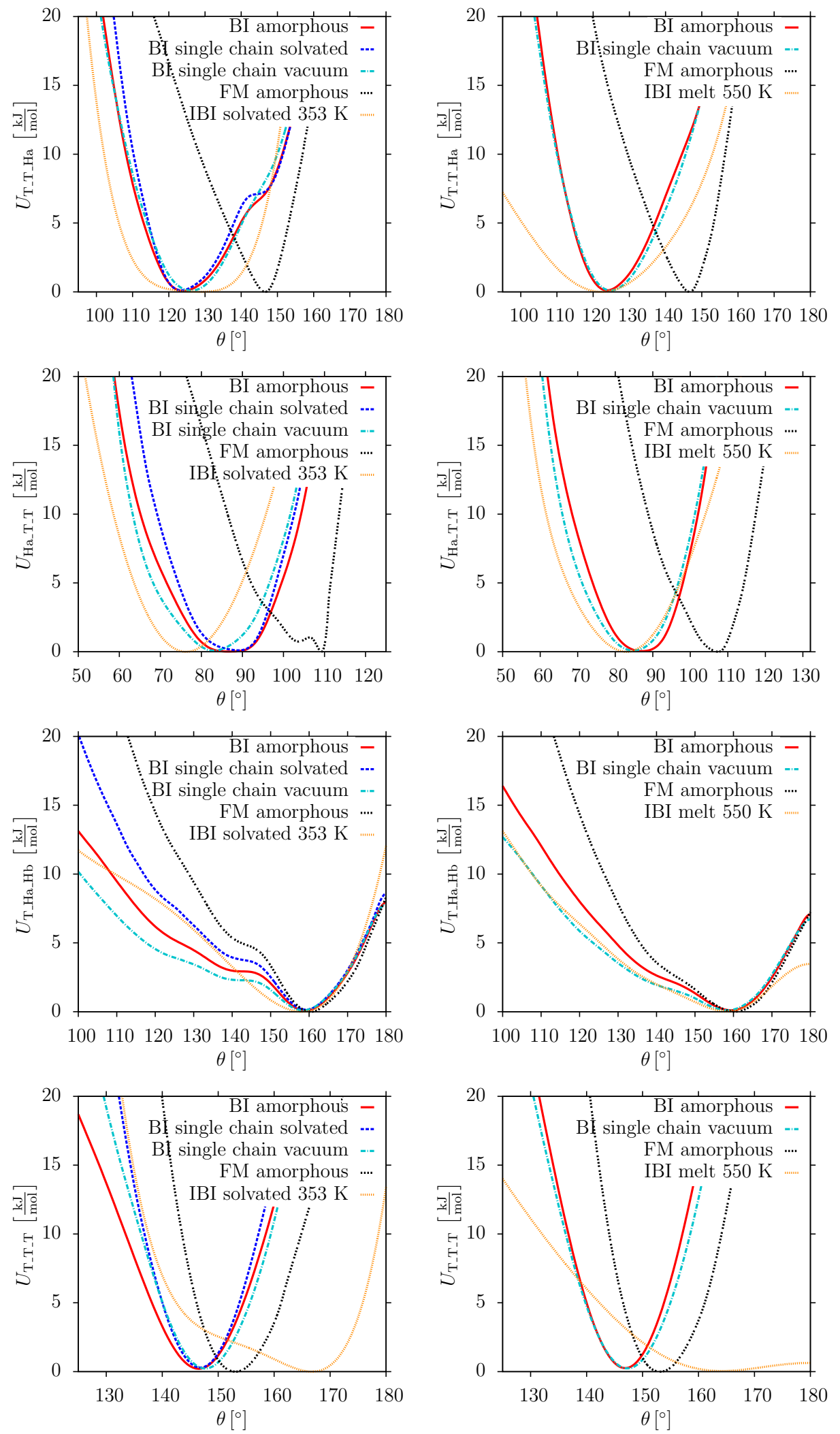

Fig. 2. Bond angle potentials parametrized at $300 \mathrm{~K}$ (left column) and $500 \mathrm{~K}$ (right column). Comparison to parametrization of $\mathrm{P} 3 \mathrm{HT}$ decamers in anisole solvent at $353 \mathrm{~K} \mathrm{[39]} \mathrm{and} \mathrm{to}$ melt of P3HT 12-mers at $550 \mathrm{~K}[37]$. 

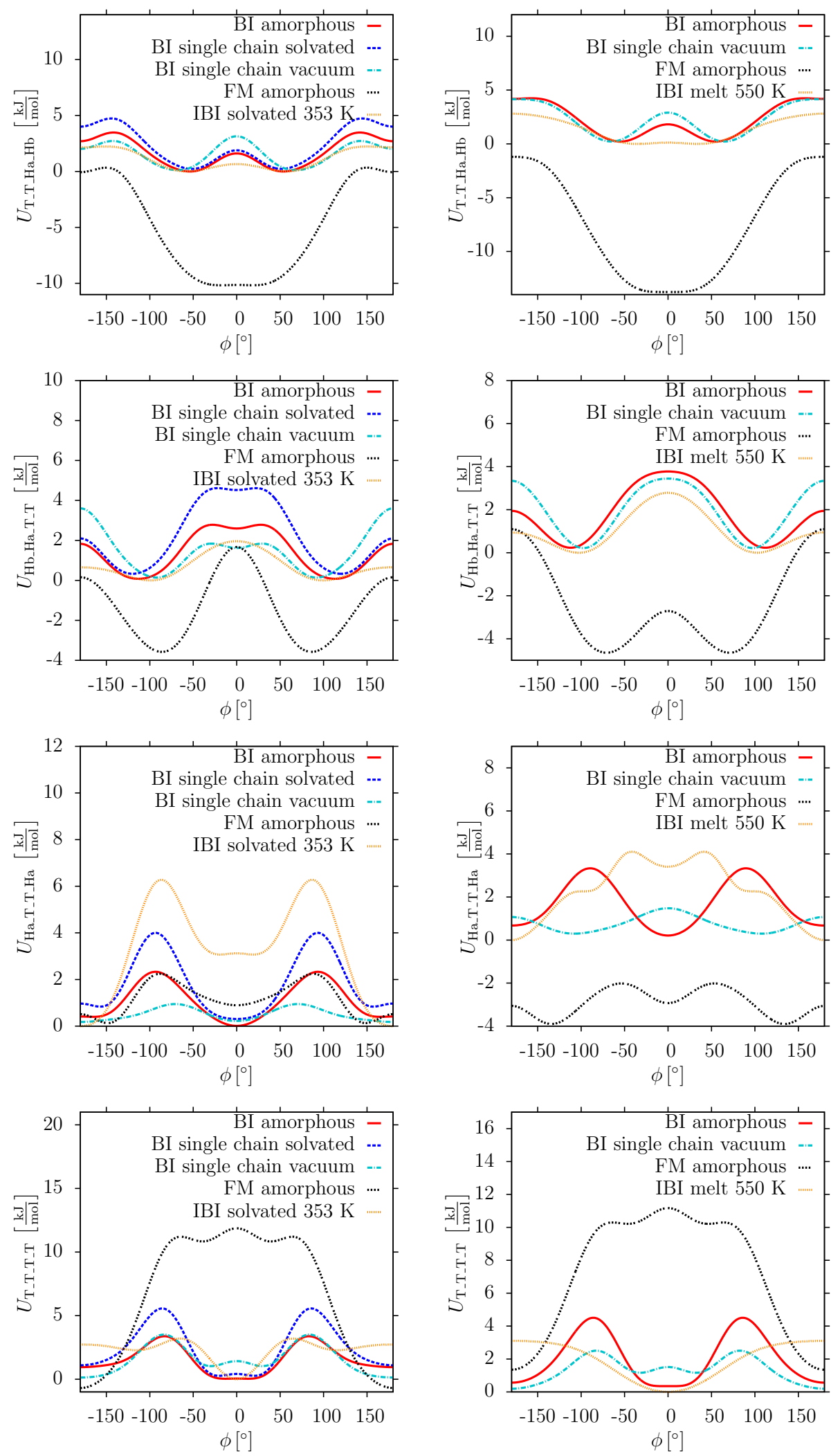

Fig. 3. Bond dihedral potentials parametrized at $300 \mathrm{~K}$ (left column) and $500 \mathrm{~K}$ (right column). Comparison to parametrization of P3HT decamers in anisole solvent at $353 \mathrm{~K}$ [39] and to melt of P3HT 12-mers at $550 \mathrm{~K}$ [37]. Here, the fits to Ryckaert-Belleman potentials, which are used in MD simulations, are shown. 
configuration where two neighboring side chains are oriented in opposing directions, which is the configuration found in the crystalline polymorphs. The BI potentials parametrized at $300 \mathrm{~K}$ show minima at $\phi=0^{\circ}$ and $\phi= \pm 180^{\circ}$ of about equal height. The same holds for the BI potential of the amorphous melt at $500 \mathrm{~K}$. This implies that the cis conformation where two neighboring side chains point in the same direction has about equal probability than the trans configuration in these systems. In both FM potentials (amorphous system at $300 \mathrm{~K}$ and $500 \mathrm{~K}$ ), the potential minima are at about $\pm 130^{\circ}-140^{\circ}$ instead of $\pm 180^{\circ}$ and the minimum at $0^{\circ}$ is less preferential. The BI potential of the single $\mathrm{P} 3 \mathrm{HT}$ chain in vacuum at $500 \mathrm{~K}$ shows a significantly different behavior with two minima at about $\pm 100^{\circ}$. The backbone dihedral potentials of previous works $[37,39], T T T T$, both, have a single minimum at $0^{\circ}$ which corresponds to a configuration where one pair of four monomers in a row is in the cis conformation meaning this is more likely than all four monomers in a row being in the trans configuration. The BI potentials of the amorphous system $(300 \mathrm{~K}$ and $500 \mathrm{~K})$ and the BI potential of the solvated chain $(300 \mathrm{~K})$ show minima at $0^{\circ}$ and $180^{\circ}$ of about equal depths. The BI potential of the single chain in vacuum shows a similar behavior. However, instead of a minimum at $0^{\circ}$, two small minima at about $\pm 40^{\circ}$ are visible. In contrast to this, the FM potentials are significantly different. They show minima only at $\pm 180^{\circ}$, implying that the atomistic forces favor a configuration where all monomers in a row are in the trans conformation, which is consistent with the FM dihedral potential $H_{a} T T H_{a}$.

To summarize, all Boltzmann inversion-based methods give roughly the same bonded potentials and are in fair agreement (taking into account slightly different mappings) to the ones of previous studies [37, 39]. The biggest differences are visible in some of the bond dihedral interactions, e.g. $H_{a} T T H_{a}$ and TTTT, of the single chain in vacuum compared to the other parametrizations. As expected, the biggest differences in the angle and dihedral potentials are observed between the FM and BI parametrizations.

\section{Nonbonded interactions}

Nonbonded interactions are parametrized using three different methods and two different systems:

1. Iterative Boltzmann inversion (IBI with pressure correction, see Appendix D for details) applied to the high temperature amorphous melt and the crystalline phase (polymorph I). In principle, IBI requires an equilibrated system at every iteration step, which is the reason for choosing the $500 \mathrm{~K}$ melt instead of the $300 \mathrm{~K}$ amorphous system. The use of the crystalline system certainly violates this requirement and is performed only out of curiosity.

2. Force matching (see Appendix C) of all interactions together, where all 11 bonded and 6 nonbonded interactions are parametrized simultaneously in the high temperature amorphous melt and the crystalline phase.

3. Force matching with exclusions [42] where the forces of the atomistic reference trajectory are recalculated, excluding all forces between atoms that belong to CG beads that are separated at most 4 repeat units from each other. This implies that the bonded interactions are excluded implicitly. The remaining forces are taken as the reference forces of the FM procedure. In this case only the 6 nonbonded interactions are parametrized and the bonded interactions are provided by the IBI parametrization.

In Fig. 4, the nonbonded CG potentials of the different CG schemes are compared to parametrizations in the amorphous melt [37] and in a mixture of P3HT monomers 

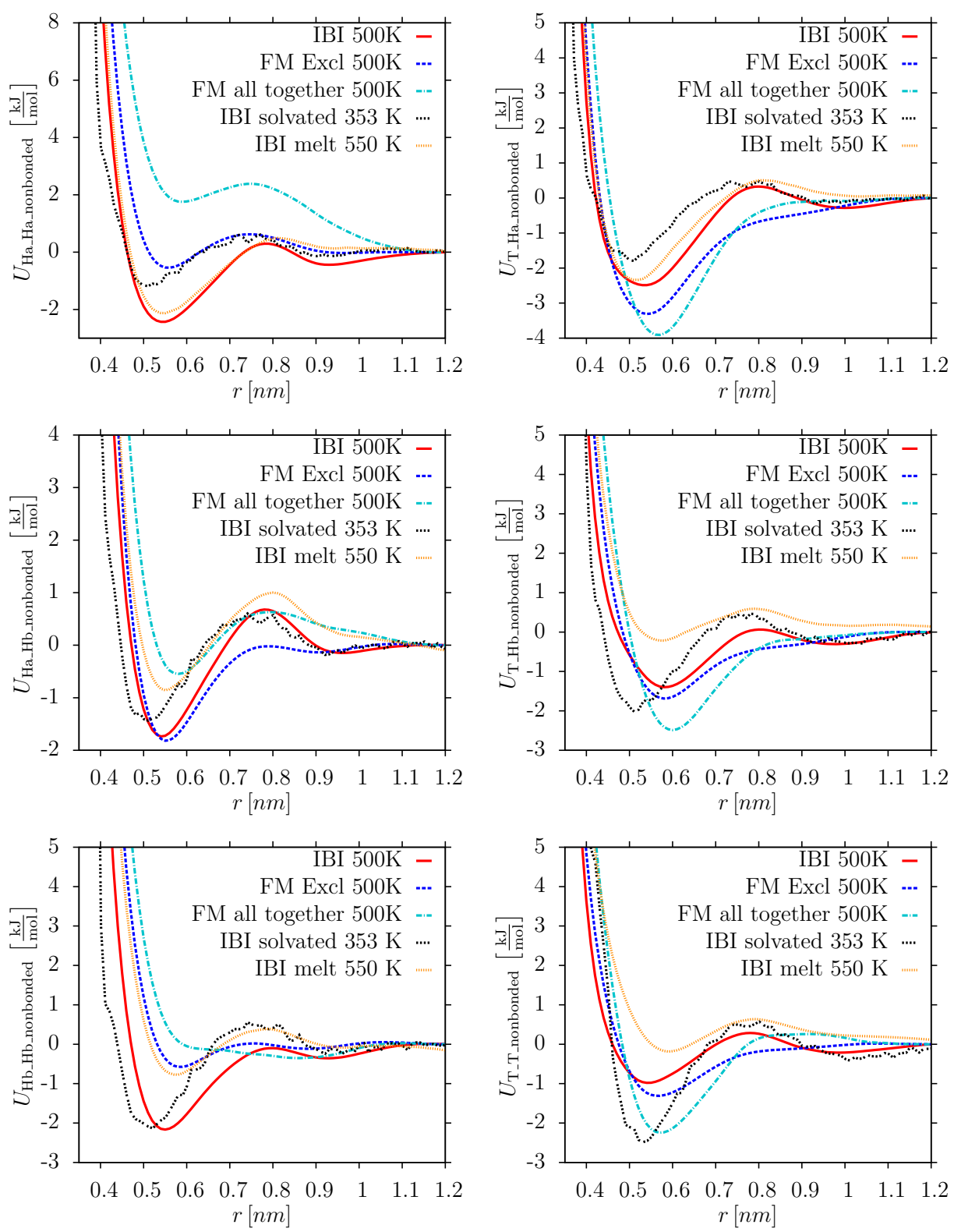

Fig. 4. Nonbonded potentials from amorphous melt of P3HT at $500 \mathrm{~K}$. Different CG methods. Comparison to parametrization of P3HT monomers in anisole solvent at $353 \mathrm{~K}$ [39] and to melt of P3HT 12-mers at $550 \mathrm{~K}$ [37].

in anisole solution [39]. One can see that in most cases the interaction potential has a repulsive core up to $0.4-0.5 \mathrm{~nm}$, followed by a first minimum at $0.5-0.6 \mathrm{~nm}$ with a well depths of $-1 \mathrm{~kJ} / \mathrm{mol}$ to $-4 \mathrm{~kJ} / \mathrm{mol}$ and a second potential minimum at $0.9-1.0 \mathrm{~nm}$ with a smaller well depths.

For the side-chain-side-chain interactions (i.e., $H_{a} H_{a}, H_{a} H_{b}$, and $H_{b} H_{b}$ ), FM of all degrees of freedom together leads to significantly more repulsive interactions compared to the other methods (FM with exclusions and IBI). No such clear distinction 

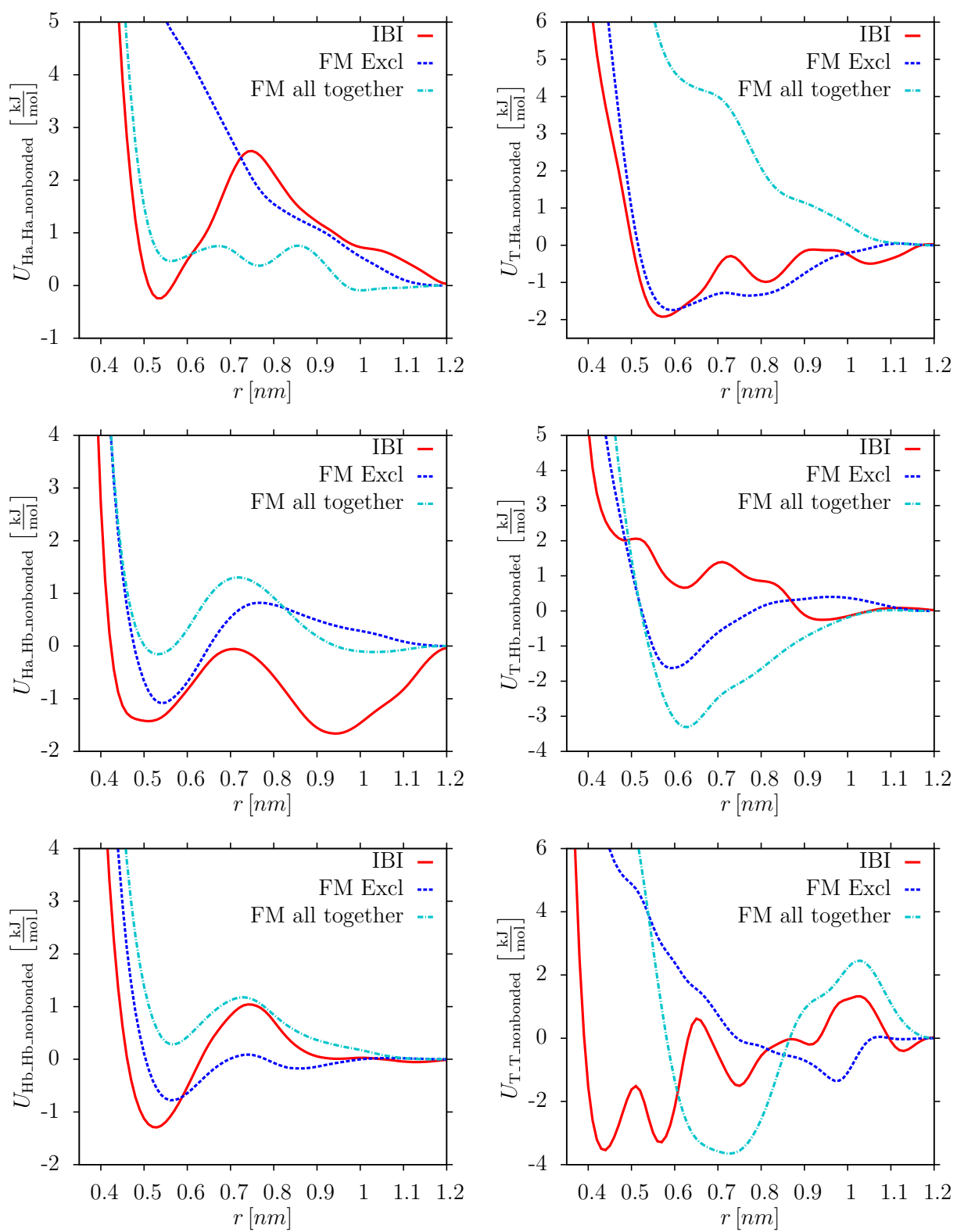

Fig. 5. Nonbonded potentials obtained in crystalline polymorph I of P3HT.

can be made for $T H_{a}, T H_{b}$ and $T T$. In fact, a similar trend has been observed for the 3 -bead CG representation of liquid hexane [42], where one of the 3 different 2-body nonbonded potentials is purely repulsive for the full FM method.

Finally, Fig. 5 shows the nonbonded CG potentials based on the crystalline polymorph I. As mentioned before, sampling of the crystalline system is not ergodic and IBI potentials have no physical meaning: the pair correlation functions in the crystalline system has several minima which are reflected in the interaction potential. On the other hand, both FM potentials will not lead to a desired lamellar molecular 
arrangement: The TT potential, which is of special interest for the stability of the crystalline polymorph, has no minima at the $\pi$-stacking distance of $0.4-0.5 \mathrm{~nm}$.

It is clear that it is not possible to foresee which of the nonbonded potentials will result in required structural and thermodynamical properties of the CG model. In the next section we will use a simple validation scheme to compare different coarse-grained parametrizations.

\section{Validation}

To assess the quality of the coarse-grained model, we have looked at several thermodynamic quantities of an amorphous melt and the crystalline polymorph I. To do this, we conduct CG MD simulations of $80 \mathrm{~ns}$ with a time step of $4 \mathrm{fs}$. The starting configurations are either mapped CG configurations of the amorphous MD simulation at $500 \mathrm{~K}$ or the crystalline phase I.

First, we evaluate the properties of the amorphous melt: For the IBI and FM with exclusions potentials NPT simulations at 1 bar are performed. For FM with all degrees of freedom parametrized together, the box expansion is too high due to the repulsiveness of the interactions. Here, an $80 \mathrm{~ns} N V T$ simulation is carried out, resulting in the average pressure of 2.34 kbar. In all cases, a Nose-Hoover thermostat is used with a damping parameter of $1 \mathrm{ps}$. In the NPT simulations, a barostat of the Parrinello-Rahman type is employed with the damping parameter of 1 ps and the compressibility parameter of $4.5 \cdot 10^{-5} \mathrm{bar}^{-1}$. In each case, the last $20 \mathrm{~ns}$ of the CG trajectories are evaluated, both for the thermodynamic and the structural quantities, and configurations are stored every 10000 time steps.

Density of an amorphous melt The density of the CG simulations is $0.87 \mathrm{~g} / \mathrm{cm}^{3}$ (IBI) and $0.76 \mathrm{~g} / \mathrm{cm}^{3}$ (FM with exclusions) in comparison to the density of the atomistic MD simulation: $0.9 \mathrm{~g} / \mathrm{cm}^{3}$. Due to the non-COM mapping of the CG beads of type $\mathrm{T}$ (see section 2), the CG mass of a P3HT chain is only about $85.8 \%$ of the atomistic one. The CG densities are scaled by this factor to compare to the atomistic ones. Having this in mind, the IBI CG densities are about $96.9 \%$ (IBI) and $85.4 \%$ (FM with exclusions) of the atomistic one. It is of course not a surprise, since IBI with pressure correction reproduces local structure by construction. This is also in good agreement with previous simulations of the amorphous melt [37], where the largest deviation between the CG and atomistic mass density is $4 \pm 2 \%$ at $650 \mathrm{~K}$ for the $\mathrm{CG}$ potential parametrized at $550 \mathrm{~K}$.

Local structure of the amorphous melt In Fig. 6, we compare the pair correlation functions of the CG simulations to the atomistic ones at $500 \mathrm{~K}$. The labels (IBI) and (FM with exclusions) refer to NPT simulations, whereas the label (FM all together) refers to the $N V T$ simulation at the atomistic system density. In general, the agreement of the CG and the atomistic curves is good. The IBI pair correlation functions practically match the atomistic ones. This is expected, as the latter are the target functions of the structure based IBI iterations. However, the short-range cutoff of the interaction potentials is $r_{\text {cut }}=1.2 \mathrm{~nm}$, both, for the atomistic and the CG potentials. Therefore, the agreement for inter-monomer distances larger than $1.2 \mathrm{~nm}$ can not be expected a priori. The largest deviations between the CG and atomistic curves is visible for the FM scheme of all interactions together, most visible in $g_{T_{-} T}(r)$. Similar observations hold for the bond length, bond angle and bond dihedral distributions. 

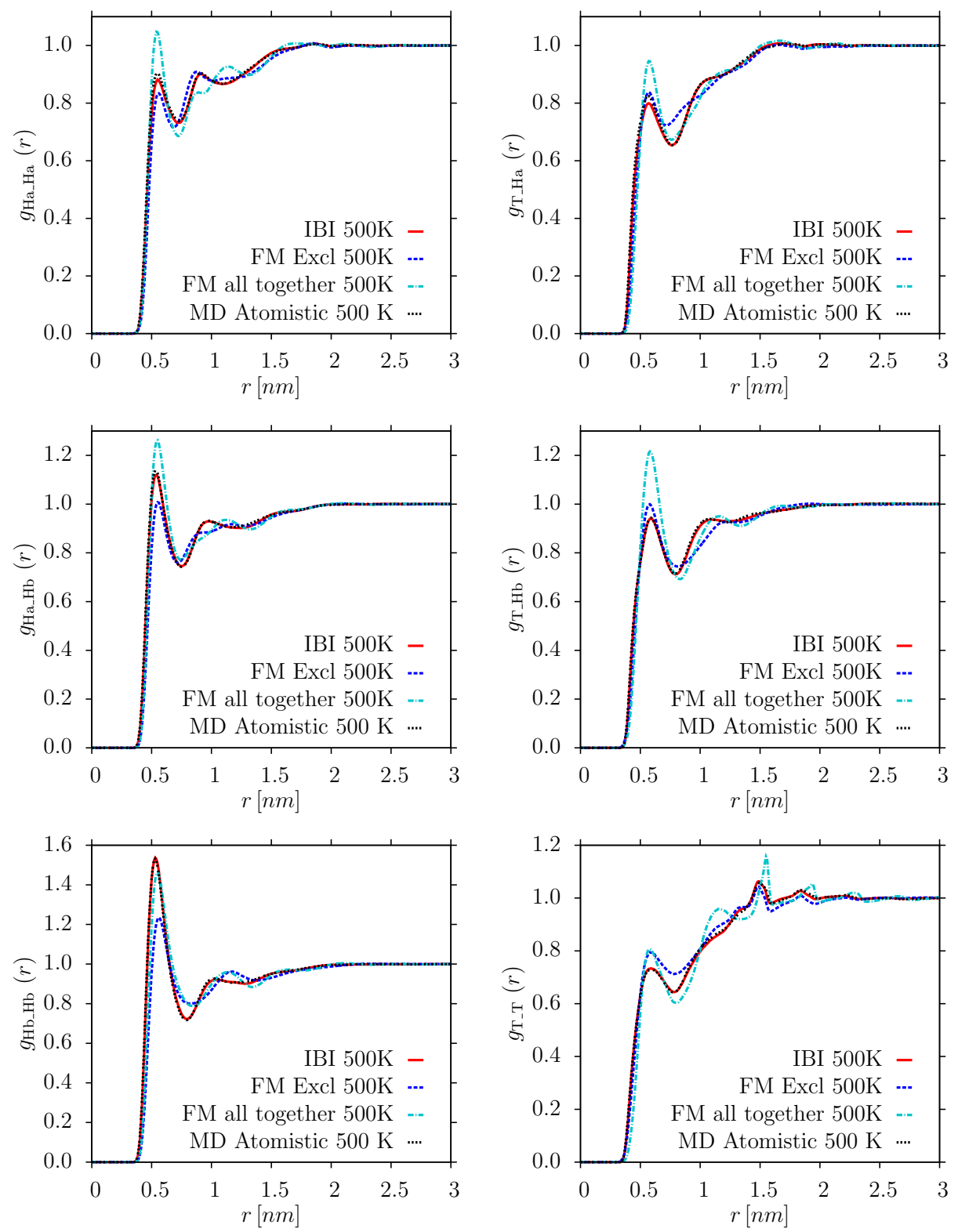

Fig. 6. Pair correlation functions of amorphous melt at $500 \mathrm{~K}$. Comparison of different CG simulations with atomistic MD simulation.

Stability of the crystalline phase In order to be able to observe a crystallization of P3HT chains from solution or from the melt in silico, it is important to have an interaction potential that keeps the crystalline phase stable. The performance of all 6 different CG models on the crystalline polymorph I is tested. NVT and NPT simulations are carried out with the CG starting configuration of the atomistic MD simulation containing 400 P3HT 20-mers. The simulation parameters are the same as in case of the CG simulations of the amorphous melt. However, the angle between the $\pi$-stacking $(y)$ and the lamellae $(x)$ directions of the simulation box is allowed 
to relax with a target stress of 1 bar. All three CG potentials of the amorphous melt derived at $500 \mathrm{~K}$ keep the lamellar ordering of the crystal structure stable, i.e. the ordering of the P3HT oligomers in $\pi$-stacks and lamellae remains intact in the CG simulation, but not the exact crystal structure of the crystalline polymorph I.

As in case of the amorphous melt, the CG parametrization from FM with all degrees of freedom parametrized together results in a too high average pressure of 5.18 kbar in the $N V T$ simulation at the atomistic box dimensions. This, again, leads to a fast box expansion in the $N P T$ simulation making a $N P T$ simulation unstable. Regarding the potentials parametrized from the crystalline phase, only the IBI potential allows for a CG simulation run where the crystalline structure does not dissolve. As already pointed out before, this is because the two CG potentials resulting from the different FM schemes do not have a potential minimum in $U_{T_{-} T}$ at the $\pi$-stacking distance of 0.4-0.5 nm (see Fig. 5).

\begin{tabular}{|c||c|c|c||c|}
\hline systems & $\begin{array}{c}\text { IBI crystalline } \\
\text { polymorph I } \\
(300 \mathrm{~K})\end{array}$ & $\begin{array}{c}\text { IBI amorphous } \\
\text { melt }(500 \mathrm{~K})\end{array}$ & $\begin{array}{c}\text { FM with } \\
\text { exclusions } \\
\text { amorphous melt } \\
(500 \mathrm{~K})\end{array}$ & MD atomistic \\
\hline \hline$L_{\mathrm{x}}[\mathrm{nm}]$ & 18.7962 & 15.7724 & 21.3148 & 16.8746 \\
\hline$L_{\mathrm{y}}[\mathrm{nm}]$ & 15.4514 & 17.0013 & 16.6898 & 15.0324 \\
\hline$L_{\mathrm{z}}[\mathrm{nm}]$ & 7.84636 & 7.8982 & 7.84628 & 8.08108 \\
\hline$\alpha_{\mathrm{yx}}\left[^{\circ}\right]$ & 80.25 & 83.13 & 73.64 & 84.74 \\
\hline
\end{tabular}

Table 2. Box dimensions of the crystalline polymorph I. Different CG schemes compared to atomistic MD simulation.

Table 2 compares the average box dimensions of the NPT simulations of the different parametrizations to the box dimensions of the atomistic simulation. The $z$-direction of the simulation box changes the least between different systems, since this is the direction along the backbones of the P3HT oligomers. Regarding the $\pi$ stacking $(y$-)direction of the simulation box, all CG simulations show a slight increase compared to the atomistic MD simulation of about $3 \%$ (IBI of crystalline polymorph I) to about $13 \%$ (IBI of melt at $500 \mathrm{~K}$ ). The largest variance of the box dimensions occurs in the $x$-direction of the simulation box.

In Fig. 7, representative snapshots for all CG potentials are shown that allow stable NPT simulations of the crystalline phase. These are compared to a CG snapshot of the crystalline polymorph I from the atomistic MD simulation. It can be clearly seen that the ordering into $\pi$-stacks and lamellae stays intact in all cases. However, the local ordering of the chains changes in the CG simulations compared to the atomistic one. The snapshot corresponding to the IBI parametrization of the crystal structure at $300 \mathrm{~K}$ (bottom right) is in the best agreement with the atomistic simulation (top left).

The local structural ordering is examined in terms of pair correlation functions, shown in Fig. 8. As expected, IBI of the crystal structure agrees the best with the target functions of the atomistic simulation. The peaks in $H_{a} H_{a}$ and $T H_{a}$ are even more pronounced than in the atomistic MD simulation. Also, the CG potentials of the amorphous melt reproduce the structure quite well. In general, the peak heights are lower and the peaks are slightly broader in the CG simulations. The crystalline ordering of the backbones is best captured with $T T$ : The first-neighbor peak corresponds to the closest distance between two thiophene rings of neighboring chains, i.e. the $\pi$-stacking distance. The peak maximum is shifted to slightly larger distances from about $0.43 \mathrm{~nm}$ (MD atomistic) to about $0.47-0.5 \mathrm{~nm}$ for the different CG potentials 

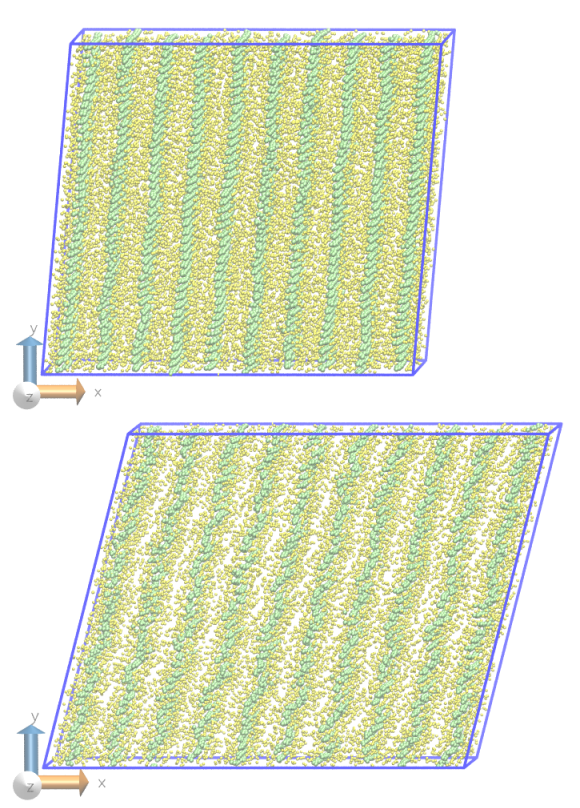
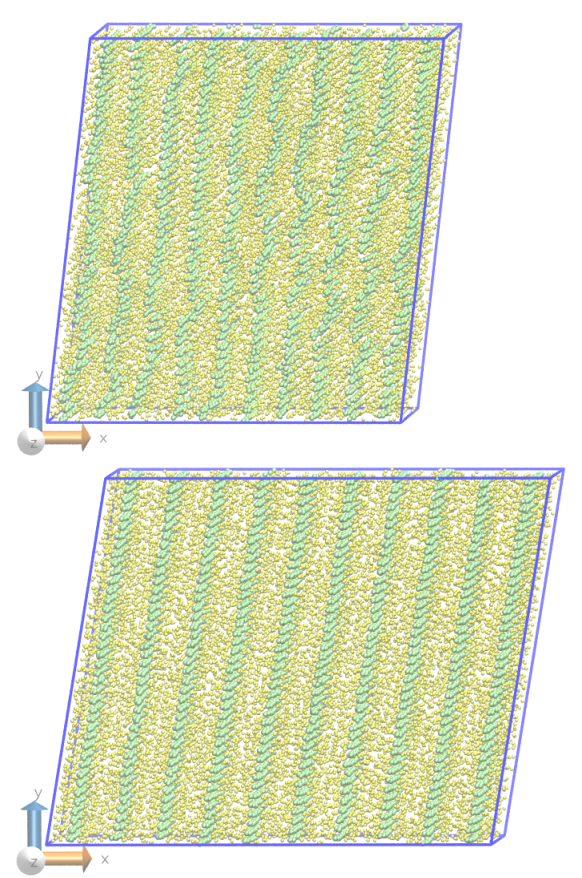

Fig. 7. Snapshots of different systems. Comparison of NPT simulations of atomistic to CG simulations. Top left: Atomistic MD. Top right: IBI of melt at $500 \mathrm{~K}$. Bottom left: FM with exclusions of melt at $500 \mathrm{~K}$. Bottom left: IBI of crystalline polymorph I at $300 \mathrm{~K}$.

of the amorphous melt at $500 \mathrm{~K}$. The second and third peak resemble the distance between the thiophene ring ( $T$-bead) of one chain and the $T$-bead displaced by one or two bonds of the next chain in the $\pi$-stacking direction. The correlation functions involving the side chains are more complicated to interpret due to the amorphous nature of the side chains in this crystalline polymorph. However, the first-neighbor peaks in $H_{a} H_{a}, H_{a} H_{b}$ and $H_{b} H_{b}$ resemble the $\pi$-stacking distance, as well. They correspond to the distance between to $\mathrm{CG}$ units of the hexyl side chains of neighboring chains in the y-direction of the simulation box.

Dihedral distributions in the crystalline phase To obtain a clearer picture of the backbone and side chain ordering, we have also examined the bond dihedral distributions. Fig. 9 depicts all relevant distributions. TTTT and $H_{a} T T H_{a}$ show one sharp maximum at $\phi= \pm 180^{\circ}$. This corresponds to two neighboring monomers being in the trans configuration where the side chains point in opposite directions. This herringbone conformation is the only occurring configuration in the crystal structure. As in case of the pair correlation functions, the peak height decreases and the peak width increases for the CG potentials parametrized in the amorphous melt at $500 \mathrm{~K}$. An additional small peak in $H_{a} T T H_{a}$ is visible at $\phi=0^{\circ}$ in all configurations, corresponding to the cis conformation of two neighboring monomers. These defects are most pronounced for the FM with exclusions potential of the $500 \mathrm{~K}$ melt in agreement with this parametrization leading to the largest deformation of the simulation box. In TTTT, an additional peak at $\phi=0^{\circ}$ is barely visible in all cases. This dihedral angle corresponds to the conformation where one pair of four monomers in a row is in the cis conformation. $T T H_{a} H_{b}$ and $H_{b} H_{a} T T$ give more insight in the conformation of the hexyl side chains. These distributions show maxima at about $\phi= \pm 60^{\circ}$ 

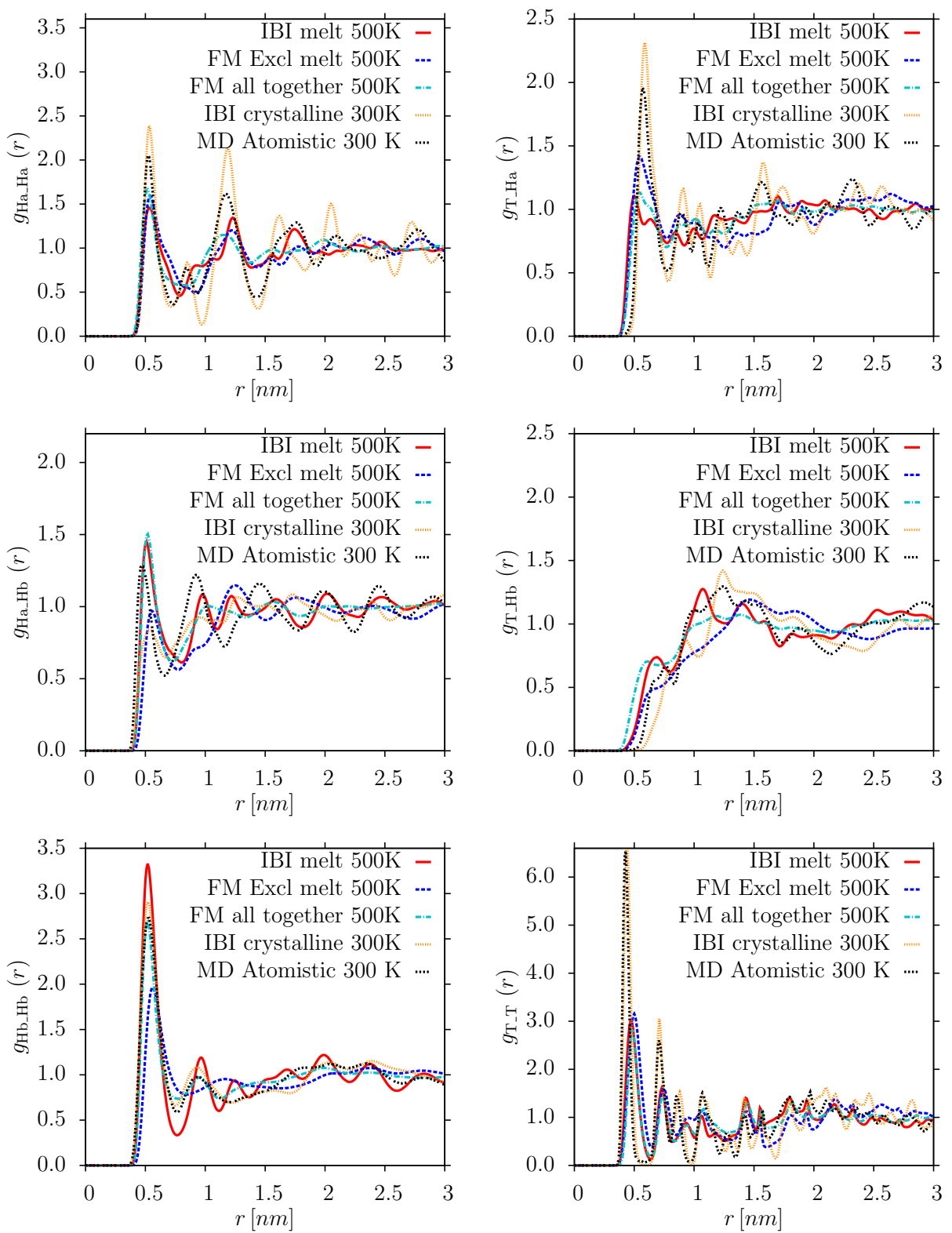

Fig. 8. Pair correlation functions of crystalline polymorph I at $300 \mathrm{~K}$. Comparison of different $\mathrm{CG}$ simulations with atomistic MD simulation.

$\left(T T H_{a} H_{b}\right)$ and about $\phi= \pm 120^{\circ}\left(H_{b} H_{a} T T\right)$ corresponding to configurations where the side chains point out of the plane determined of the backbones by about $60^{\circ}$. This is due to the $\mathrm{CH}_{3}$ bond. The atomistic MD distributions show additional peaks at $\phi= \pm 180^{\circ}\left(T T H_{a} H_{b}\right)$ and $\phi=0^{\circ}\left(H_{b} H_{a} T T\right)$ which are not present in the CG distributions. These correspond to configurations where the side chains stick out in the plane fixed by the backbones. In case of the atomistic MD simulations these configurations are mainly stabilized by the nonbonded interactions. The difference in the CG simulations can be understood when looking at the bond dihedral potentials de- 

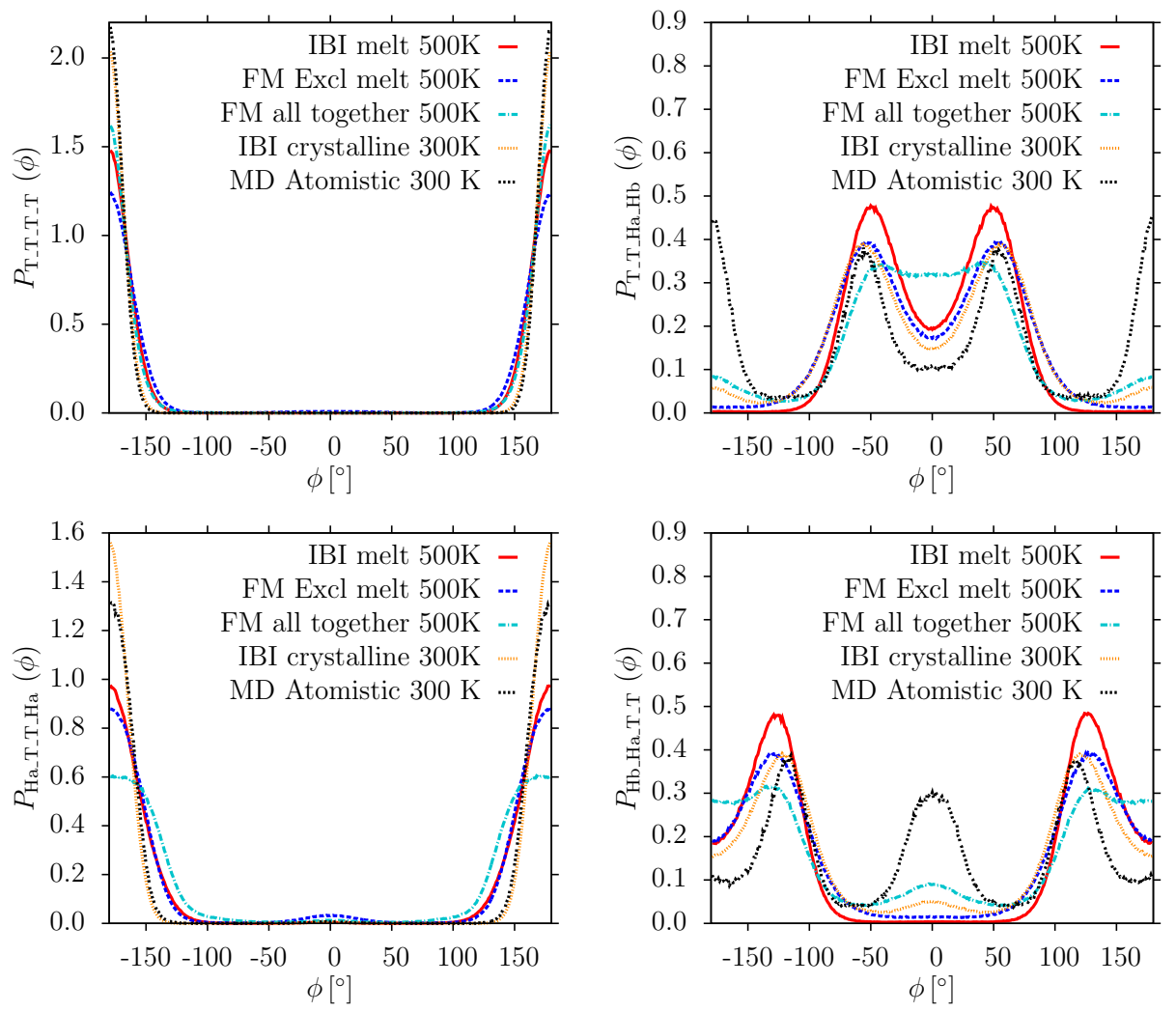

Fig. 9. Distributions of bond dihedral angles of crystalline polymorph I at $300 \mathrm{~K}$. Comparison of different $\mathrm{CG}$ simulations with atomistic MD simulation.

picted in Fig. 3. The bonded potentials used in the CG simulations are the ones from the amorphous system which lack the additional peaks at $\phi= \pm 180^{\circ}\left(T T H_{a} H_{b}\right)$ and $\phi=0^{\circ}\left(H_{b} H_{a} T T\right)$. In case of $H_{a} T T H_{a}$ and $T T T T$, the additional potential maxima at $\phi=0^{\circ}$ in the amorphous system are practically not sampled when starting from the starting configuration of the crystalline polymorph I due to steric hindrance.

\section{Conclusions}

In this work, we developed a set of coarse-grained force fields for the P3HT polymer. To parametrize the models, Boltzmann inversion, iterative Boltzmann inversion with simple pressure correction, force matching of all degrees of freedom together, and force matching with excluded bonded interactions have been used.

The bonded interactions, namely bond length, bond angle and bond dihedral interactions, have been parametrized based on the simulation of a single chain in vacuum (at $300 \mathrm{~K}$ and $500 \mathrm{~K}$ ), a single chain solvated in DCB solvent (at $300 \mathrm{~K}$ ) and an amorphous configuration (melt) (at $300 \mathrm{~K}$ and $500 \mathrm{~K}$ ). The nonbonded interactions have been evaluated for the atomistic configurations of an amorphous melt at $500 \mathrm{~K}$ and the crystalline polymorph I at $300 \mathrm{~K}$. In all cases, nonbonded interactions have been represented by tabulated two-body potentials. 
The CG force fields have been validated by: (i) reproduction of the properties of the amorphous melt (density and radial distribution functions) and (ii) preservation of the experimentally known crystal structure of the crystalline polymorph I.

All CG force fields parametrized in the amorphous melt at $500 \mathrm{~K}$ are capable of reproducing the structural properties of the melt. The IBI parametrization with pressure correction leads to a density of $96.9 \%$ of the atomistic system, while the FM scheme with exclusions results in a density of $85.4 \%$. The FM applied to all degrees of freedom together leads to too small system densities.

All CG force fields parametrized in the amorphous melt at $500 \mathrm{~K}$ are also capable of preserving the lamellar ordering and $\pi$-stacking of the crystalline polymorph I. However, the local ordering of the chains, $\pi$-distances and interlamellar separations change: The $\pi$-stacking distance slightly increases, whereas the lamellar spacing slightly decreases for the IBI potential and significantly increases for the potential from FM with exclusions. Among the CG potentials parametrized in the crystalline phase, only the IBI potential can keep the crystal structure stable. However, this potential is unphysical and not transferable to other, e. g., amorphous systems.

The key result of this work is that the coarse-grained potentials, parametrized in the amorphous state of $\mathrm{P} 3 \mathrm{HT}$, also preserve, to a certain degree, its lamellar ordering. Surprisingly, IBI with a simple pressure correction provides the best model in this respect. The best force matching results are obtained when only the nonbonded interactions are parametrized, i.e. by excluding all interatomic forces contributing to the coarse-grained bonded interactions.

\section{Acknowledgments}

This work was in part supported by the BMBF grants MEDOS (FKZ 03EK3503B), MESOMERIE (FKZ 13N10723), and InterPhase (FKZ 13N13661). The project has received funding from the NMP-20-2014 - "Widening materials models" program under Grant Agreement No. 646259 (MOSTOPHOS). We acknowledge financial support by DFG through the collaborative research center TRR 146 .

\section{A Simulation details}

All atomistic molecular dynamics (MD) simulations of the crystalline polymorph I [21], the amorphous system, a single P3HT chain in dichlorobenzene (DCB) solvent and single chain in vacuum are performed using the GROMACS package [43] (version 5.1) with a time step of $1 \mathrm{fs}$. The atomistic interaction potential is a modified version of the OPLS-AA [3, 44] force field [30]. In case of the simulation of the solvated P3HT chain, the DCB force field parameters are also taken from the OPLS force field.

All simulations are performed using $100 \%$ regioregular chains with 20 repeat units, namely a thiophene ring with the hexyl side chain. In each case, the system is first equilibrated in a $N P T$ run at constant external pressure. Afterwards, a $N V T$ production run is carried out at the mean box dimensions of the preceding NPT run. In all cases, a Nose-Hoover thermostat $[45,46]$ is used with a damping parameter of $1 \mathrm{ps}$, meaning the temperature is relaxed to the target temperature in a time span of 1000 time steps. For constant pressure simulations, a barostat of the Parrinello-Rahman type $[47,48]$ is employed with a target pressure of $p_{\text {target }}=1$ bar which is the atmospheric pressure. The damping parameters are set to $1 \mathrm{ps}$ and the compressibility parameters are set to the one of water, namely $4.5 \cdot 10^{-5} 1 / \mathrm{bar}$.

The crystalline setup is taken from Ref. [30], namely a supercell containing 400 chains (10 lamellae and 20 chains in the $\pi$-stacking direction). The NPT equilibration 
at $300 \mathrm{~K}$ is done for $15 \mathrm{~ns}$. In this case, in addition to the box dimension also the angle between the $\pi$-stacking $(y)$ and the lamellae $(x)$ direction of the simulation box is allowed to relax with a target stress of 1 bar. Again, the damping and compressibility parameters are $1 \mathrm{ps}$ and $4.5 \cdot 10^{-5} 1 / \mathrm{bar}$. The mean box dimensions of the last $5 \mathrm{~ns}$ of the production run are: $L_{x}=16.8746 \mathrm{~nm}, L_{y}=15.0324 \mathrm{~nm}, L_{z}=8.08108 \mathrm{~nm}$ and $\alpha_{y x}=84.74^{\circ}$. This corresponds to a density of $\rho=1.08 \mathrm{~g} / \mathrm{cm}^{3}$. The $N V T$ production run is carried out for $1 \mathrm{~ns}$.

Amorphous systems are studied at two different temperatures, namely $300 \mathrm{~K}$ and $500 \mathrm{~K}$. As mentioned in the introduction, the first temperature is about the glass transition temperature and the second one above the melting temperature of the crystalline phase in the liquid state [32]. The systems are prepared as follows: First, the crystalline system is heated up to $750 \mathrm{~K}$ in $100 \mathrm{ps}$ and then equilibrated at $750 \mathrm{~K}$ for another $3627 \mathrm{ps}$ until the originial crystal structure has dissolved. Afterwards, the system box is replicated fourfold in $z$-direction and now contains 1600 P3HT chains. To obtain the amorphous system at $300 \mathrm{~K}$, this configuration is quenched down to 300 in $100 \mathrm{ps}$ and annealed at $300 \mathrm{~K}$ for about $60.6 \mathrm{~ns}$. To prepare the amorphous structure at $500 \mathrm{~K}$ the same configuration is quenched down from $750 \mathrm{~K}$ to 300 in $100 \mathrm{ps}$ and annealed at $500 \mathrm{~K}$ for about $12.4 \mathrm{~ns}$. In contrast to all other simulations, these runs are carried out with a Berendsen thermostat and barostat [49] with time constants of $1 \mathrm{ps}(N V T$ and $N P T)$ for the equilibration at $750 \mathrm{~K}$, of $1 \mathrm{ps}(N V T)$ and $0.5 \mathrm{ps}(N P T)$ for the quench and annealing at $300 \mathrm{~K}$ and of $0.1 \mathrm{ps}(N V T)$ and $0.5 \mathrm{ps}(N P T)$ for the simulation run at $500 \mathrm{~K}$. The resulting configurations are taken from [50]. Next, the equilibration at $300 \mathrm{~K}$ is continued with a $4.1 \mathrm{~ns}$ run with the Nose-Hoover thermostat and barostat with the same parameters as for the crystalline system. The $500 \mathrm{~K} \mathrm{NPT}$ simulation is continued for another $12 \mathrm{~ns}$. It should be noted that at $300 \mathrm{~K}$ the P3HT chains show nearly now diffusion. Therefore, at least the intermediate range structure of the $300 \mathrm{~K}$ configuration resembles the structure at a higher temperature. At $500 \mathrm{~K}$, however, the amorphous system can be equilibrated at the timescale of the NPT simulation. The mean box dimensions of the last $3.1 \mathrm{~ns}$ of the production run at $300 \mathrm{~K}$ are: $L_{x}=19.6108 \mathrm{~nm}, L_{y}=22.8062 \mathrm{~nm}, L_{z}=18.9935 \mathrm{~nm}$ and $\alpha_{y x}=87^{\circ}$. At $500 \mathrm{~K}$, the box dimensions are averaged over the last $10 \mathrm{~ns}: L_{x}=20.947 \mathrm{~nm}$, $L_{y}=24.2387 \mathrm{~nm}, L_{z}=19.4284 \mathrm{~nm}$. In both cases, the angle between the $x$ and the $y$ axis is fixed to $\alpha_{y x}=87^{\circ}$. The dimensions correspond to densities of $\rho=1.03953 \mathrm{~g} / \mathrm{cm}^{3}$ $(300 \mathrm{~K})$ and $\rho=0.895231 \mathrm{~g} / \mathrm{cm}^{3}(500 \mathrm{~K})$. The $N V T$ production runs are of the length of $500 \mathrm{ps}(300 \mathrm{~K})$ and $1 \mathrm{~ns}(500 \mathrm{~K})$.

In addition to these configurations with 400 and 1600 oligomers, two different kinds of single chain simulations are carried out. They are set up to sample the conformations within the P3HT chain. Again, regioregular chains with 20 repeat units are used. In one case, the chain is solvated in 8000 DCB molecules. Here, a cubic simulation box is used. After an NPT equilibration of $2000 \mathrm{ps}$ at $300 \mathrm{~K}$, a production run of $1000 \mathrm{ps}$ is carried out at the same temperature. The mean box dimensions of the $N P T$ run are $L_{x}=L_{y}=L_{z}=11.4238 \mathrm{~nm}$. This refers to a density of $\rho=1.31 \mathrm{~g} / \mathrm{cm}^{3}$. In the other case, the chain conformations of a single chain in vacuum are studied. To prevent backfolding of the polymer chain, all non-bonded interactions between atoms are excluded that are more than 4 repeat units apart along the backbone of the chain. In this case the box size is fixed to $L_{x}=L_{y}=L_{z}=20 \mathrm{~nm}$ without periodic boundary conditions and the chain conformations are sampled in a $N V T$ run of $50000 \mathrm{ps}$.

The interactions between the CG sites can be divided into bonded and non-bonded interactions. In this work, the bonded interactions are parametrized by two different methods, namely Boltzmann inversion (BI) [16] and force matching (FM) [8, 5]. The nonbonded interactions are parametrized by iterative Boltzmann inversion (IBI) [6] and force matching (FM). In all cases, a cutoff of $r_{\text {cut }}=1.2 \mathrm{~nm}$ is used for the nonbonded potentials. This is consistent with the short-range cutoff of the atomistic 
force field. In addition, nonbonded interactions are only taken into account between CG beads that are separated more than four repeat units from each other.

\section{B Boltzmann inversion}

The general idea of Boltzmann inversion (BI) is that in a canonical ensemble, the partition function of independent degrees of freedom factorizes. For each independent variable $q$ it has the following form: $P_{q}(q) \propto \exp [-\beta U(q)], \beta=1 / k_{\mathrm{B}} T$, and can be inverted to obtain the $\mathrm{CG}$ potential of this variable, namely:

$$
U(q)=-k_{\mathrm{B}} T \ln P_{q}(q)+\text { const }, \quad q=r, \theta, \phi .
$$

It is a potential of mean force and, due to construction, neglects all correlations between different degrees of freedom. In practice, this usually works well for the parametrization of bonded interactions. It should be noted that the histograms of bonds $H_{r}(r)$, angles $H_{\theta}(\theta)$ and dihedrals $H_{\phi}(\phi)$ have to be normalized [7] in order to get the correct distribution functions: $P_{r}(r)=\frac{H_{r}(r)}{4 \pi r^{2}}, P_{\theta}(\theta)=\frac{H_{\theta}(\theta)}{\sin (\theta)}$ and $P_{\phi}(\phi)=H_{\phi}(\phi)$. In the first place, all bonded interactions are tabulated and a simple smoothing is performed. Second, the dihedral interactions are represented by analytical functions of the Ryckaert-Belleman type: $\sum_{i=0}^{5} c_{i} \cos \left(180^{\circ}-\phi\right)$. The coefficients are determined by a least squares fit to the tabulated potentials.

\section{Force matching}

The force matching (FM) or multiscale coarse-graining (MS-CG) in its most general description leads to a many-body potential of mean force that yields an equilibrium distribution of the CG degrees of freedom which is consistent with the atomistic system from which this potential has been derived [5]. In practice, this potential of mean force has to be approximated by means of a set of basis functions. In the current implementation of the force matching routine of the VOTCA package [42] these basis functions are cubic splines. The procedure is as follows:

The reference forces on the coarse-grained beads can be written in the following form:

$$
f_{i}^{\mathrm{ref}}=M_{i} \sum_{\alpha \in i} \frac{w_{\alpha} f_{\alpha}}{m_{\alpha}} .
$$

Here, $M_{i}$ is the mass of the CG bead (see equation (2)). The forces on the CG beads are a weighted sum of the atomistic forces on all atoms belonging to the coarse-grained bead with mapping coefficients according to equation (1). The CG representation of the force is then determined by solving the following system of equations:

$$
f_{i l}^{\mathrm{CG}}\left(g_{1}, \ldots, g_{M}\right)=f_{i l}^{\mathrm{ref}}, \quad i=1, \ldots, N, \quad l=1, \ldots, L .
$$

The coefficients $g_{1}, \ldots, g_{M}$ are the spline coefficients of the set of cubic splines necessary to represent all interactions, $N$ is the number of CG beads and $L$ the number of simulation snapshots analyzed in parallel. The condition $M<N \times L$ has to be fulfilled, in order to get a unique solution. In practice, $M>N \times L$ and the problem is overdetermined. Employing (cubic) splines as basis functions ensures that the set of equations (5) is a set of linear equations. The VOTCA implementation of the cubic splines and the calculation of the bonded (bond, angle) and nonbonded interaction are described in detail in [7]. In this work, the FM routine has been extended to calculate 
tabulated dihedral interactions. In this course, additional restraints are implemented. The first one guarantees a periodic force and the second one implies that the average dihedral force is equal to zero. In all cases (bonded and nonbonded), the interaction potentials are determined from the forces by numerical integration. For the nonbonded interactions, before the numerical integration, first, a simple smoothing is carried out and, second, the tabulated 2-body forces are multiplied by an analytic function of the form:

$$
f_{\text {switch }}(r)=\cos \left(\frac{\pi}{2} \frac{r-r_{\mathrm{sm}}}{r_{\mathrm{cut}}-r_{\mathrm{sm}}}\right) .
$$

This is done for all distances greater than $r_{\mathrm{sm}}=1.0$ to ensure a smooth decay to zero at the short range cutoff of $r_{\text {cut }}=1.2 \mathrm{~nm}$.

\section{Iterative Boltzmann inversion}

Iterative Boltzmann inversion (IBI) is an extension of the simple Boltzmann inversion. Here, the interaction potential iteratively refined, according to the following update scheme:

$$
U^{(n+1)}=U^{(n)}+\alpha \Delta U^{(n)}, \Delta U^{(n)}=k_{\mathrm{B}} T \ln \frac{P^{(n)}}{P_{\text {ref }}} .
$$

This is an empirical scheme and reaches convergence as soon as the distribution function $P^{(n)}$ matches the reference distribution function $P_{\text {ref }}$. In this work, IBI is only used for the nonbonded intaractions and the distribution function refers to the pair correlation function $g(r)$. The choice of a scaling parameter $\alpha<1$ can help to stabilize the algorithm. As the target function(s) of IBI is/are the structural quantities, one can in general not expect to obtain a $\mathrm{CG}$ model that reproduces thermodynamical quantities correctly at the same time. It has been found [6] that a simple pressure correction in terms of adding a linear inter-particle correlation function can help finding a potential that, at least, yields an approximately correct system density:

$$
\Delta U(r)=A\left(1-\frac{r}{r_{\text {cut }}}\right),
$$

$A=-\operatorname{sgn}(\Delta p) 0.1 k_{\mathrm{B}} T \min (1, f \Delta p)$. Here, $\Delta p=p_{\mathrm{i}}-p_{\text {target }}$ and $f$ is a scaling factor. Here, this post-processing is applied at every second step and $\alpha=0.1$ and $f=0.001$ are chosen in all cases.

\section{References}

1. J. Zinn-Justin, Quantum Field Theory and Critical Phenomena, 4th edn. (Clarendon Press, Oxford : New York, 2002)

2. A. Jansen, Computer Physics Communications 86(1-2), 1 (1995)

3. W.L. Jorgensen, J. Tirado-Rives, J. Am. Chem. Soc. 110(6), 1657 (1988)

4. M. Moral, W.J. Son, J.C. Sancho-García, Y. Olivier, L. Muccioli, J. Chem. Theory Comput. 11(7), $3383(2015)$

5. W.G. Noid, J.W. Chu, G.S. Ayton, V. Krishna, S. Izvekov, G.A. Voth, A. Das, H.C. Andersen, J. Chem. Phys. 128(24), 244114 (2008)

6. D. Reith, M. Pütz, F. Müller-Plathe, Journal of computational chemistry 24(13), $1624(2003)$

7. V. Rühle, C. Junghans, A. Lukyanov, K. Kremer, D. Andrienko, J. Chem. Theory. Comput. 5(12), 3211 (2009) 
8. S. Izvekov, M. Parrinello, C.J. Burnham, G.A. Voth, J. Chem. Phys. 120(23), $10896(2004)$

9. M.S. Shell, J. Chem. Phys. 129(14), 144108 (2008)

10. J.F. Rudzinski, W.G. Noid, The European Physical Journal Special Topics 224(12), 2193 (2015)

11. L. Larini, L. Lu, G.A. Voth, J. Chem. Phys. 132(16), 164107 (2010)

12. H. Wang, C. Junghans, K. Kremer, Eur. Phys. J. E 28(2), 221 (2009)

13. C.F. Abrams, K. Kremer, Macromolecules 36(1), 260 (2003)

14. S. Leon, N. van der Vegt, L. Delle Site, K. Kremer, Macromolecules 38(19), 8078 (2005)

15. P. Gemünden, C. Poelking, K. Kremer, D. Andrienko, K.C. Daoulas, Macromolecules 46(14), 5762 (2013)

16. W. Tschöp, K. Kremer, J. Batoulis, T. Bürger, O. Hahn, Acta Polymer 49, 61 (1998)

17. C. Poelking, K. Daoulas, A. Troisi, D. Andrienko, in P3HT Revisited - From Molecular Scale to Solar Cell Devices, vol. 265, ed. by S. Ludwigs (Springer Berlin Heidelberg, Berlin, Heidelberg, 2014), pp. 139-180

18. R.D. McCullough, R.D. Lowe, J. Chem. Soc., Chem. Commun. (1), 70 (1992)

19. M.T. Dang, L. Hirsch, G. Wantz, Adv. Mater. 23(31), 3597 (2011)

20. G. Li, V. Shrotriya, J. Huang, Y. Yao, T. Moriarty, K. Emery, Y. Yang, Nat. Mater. 4(11), $864(2005)$

21. T.J. Prosa, M.J. Winokur, R.D. McCullough, Macromolecules 29(10), 3654 (1996)

22. Y. Yuan, J. Zhang, J. Sun, J. Hu, T. Zhang, Y. Duan, Macromolecules 44(23), $9341(2011)$

23. D. Dudenko, A. Kiersnowski, J. Shu, W. Pisula, D. Sebastiani, H.W. Spiess, M.R. Hansen, Angew. Chem. Int. Edit. 51(44), 11068 (2012)

24. Z. Wu, A. Petzold, T. Henze, T. Thurn-Albrecht, R.H. Lohwasser, M. Sommer, M. Thelakkat, Macromolecules 43(10), 4646 (2010)

25. K.J. Ihn, J. Moulton, P. Smith, J. Polym. Sci. Part B Polym. Phys. 31(6), 735 (1993)

26. S. Samitsu, T. Shimomura, S. Heike, T. Hashizume, K. Ito, Macromolecules 41(21), 8000 (2008)

27. W.D. Oosterbaan, V. Vrindts, S. Berson, S. Guillerez, O. Douhéret, B. Ruttens, J. D'Haen, P. Adriaensens, J. Manca, L. Lutsen, D. Vanderzande, J. Mater. Chem. 19(30), 5424 (2009)

28. J.D. Roehling, I. Arslan, A.J. Moulé, J. Mater. Chem. 22(6), 2498 (2012)

29. S. Berson, R. De Bettignies, S. Bailly, S. Guillerez, Adv. Funct. Mater. 17(8), $1377(2007)$

30. C. Poelking, D. Andrienko, Macromolecules 46(22), 8941 (2013)

31. V. Marcon, G. Raos, J. Phys. Chem. B 108(46), 18053 (2004)

32. R.S. Bhatta, Y.Y. Yimer, D.S. Perry, M. Tsige, J. Phys. Chem. B 117(34), 10035 (2013)

33. D. Curcó, C. Alemán, J. Comput. Chem. 28(10), 1743 (2007)

34. K. Do, D.M. Huang, R. Faller, A.J. Moulé, Phys. Chem. Chem. Phys. 12(44), $14735(2010)$

35. O. Alexiadis, V.G. Mavrantzas, Macromolecules 46(6), 2450 (2013)

36. C.K. Lee, C.W. Pao, C.W. Chu, Energy \& Environmental Science 4(10), 4124 (2011)

37. D.M. Huang, R. Faller, K. Do, A.J. Moulé, J. Chem. Theory. Comput. 6(2), 526 (2010)

38. D.M. Huang, A.J. Moule, R. Faller, Fluid Phase Equilibria 302(1-2), 21 (2011)

39. K.N. Schwarz, T.W. Kee, D.M. Huang, Nanoscale 5(5), 2017 (2013) 
40. E. Jankowski, H.S. Marsh, A. Jayaraman, Macromolecules 46(14), 5775 (2013)

41. J.A. Merlo, C.D. Frisbie, J. Phys. Chem. B 108(50), 19169 (2004)

42. V. Rühle, C. Junghans, Macromolecular Theory and Simulations 20(7), 472 (2011)

43. M.J. Abraham, T. Murtola, R. Schulz, S. Páll, J.C. Smith, B. Hess, E. Lindahl, SoftwareX 1-2, 19 (2015)

44. W.L. Jorgensen, J. Tirado-Rives, J. Comput. Chem. 26(16), 1689 (2005)

45. S. Nosé, Molecular Physics 52(2), 255 (1984)

46. W.G. Hoover, Phys. Rev. A 31(3), 1695 (1985)

47. M. Parrinello, A. Rahman, J. Appl. Phys. 52(12), 7182 (1981)

48. S. Nosé, M. Klein, Molecular Physics 50(5), 1055 (1983)

49. H.J. Berendsen, J.v. Postma, W.F. van Gunsteren, A. DiNola, J.R. Haak, The Journal of chemical physics 81(8), 3684 (1984)

50. P. Gemünden, C. Poelking, K. Kremer, K. Daoulas, D. Andrienko, Macromol. Rapid Commun. 36(11), 1047 (2015) 